\title{
BMJ Open Epidemiology of injuries, treatment (costs) and outcome in burn patients admitted to a hospital with or without dedicated burn centre (Burn-Pro): protocol for a multicentre prospective observational study
}

Esther MM Van Lieshout, ${ }^{1}$ Daan T Van Yperen, ${ }^{1,2}$ Margriet E Van Baar, ${ }^{3,4}$ Suzanne Polinder, ${ }^{4}$ Doeke Boersma, ${ }^{5}$ Anne YMVP Cardon, ${ }^{6}$ Piet AR De Rijcke, ${ }^{7}$ Marc Guijt, ${ }^{8}$ Taco MAL Klem, ${ }^{9}$ Koen WW Lansink, ${ }^{10}$ Akkie N Ringburg, ${ }^{11}$ Maarten Staarink, ${ }^{12}$ Leon Van de Schoot, ${ }^{13}$ Alexander H Van der Veen, ${ }^{14}$ Floortje C Van Eijck, ${ }^{15}$ Percy V Van Eerten, ${ }^{16}$ Paul A Vegt, ${ }^{17}$ Dagmar I Vos, ${ }^{18}$ Marco Waleboer, ${ }^{19}$ Michael HJ Verhofstad, ${ }^{1}$ Cornelis H Van der Vlies ${ }^{1,2}$

To cite: Van Lieshout EMM, Van Yperen DT, Van Baar ME, et al. Epidemiology of injuries, treatment (costs) and outcome in burn patients admitted to a hospital with or without dedicated burn centre (Burn-Pro): protocol for a multicentre prospective observational study. BMJ Open 2018;8:e023709. doi:10.1136/ bmjopen-2018-023709

- Prepublication history for this paper is available online. To view these files, please visit the journal online (http://dx.doi. org/10.1136/bmjopen-2018023709).

EMMVL and DTVY contributed equally.

Received 19 April 2018 Revised 21 August 2018 Accepted 12 October 2018

\section{Check for updates}

(C) Author(s) (or their employer(s)) 2018. Re-use permitted under CC BY-NC. No commercial re-use. See rights and permissions. Published by BMJ.

For numbered affiliations see end of article.

\section{Correspondence to} Professor Esther MM Van Lieshout;

e.vanlieshout@erasmusmc.nl

\section{ABSTRACT}

Introduction The Emergency Management of Severe Burns (EMSB) referral criteria have been implemented for optimal triaging of burn patients. Admission to a burn centre is indicated for patients with severe burns or with specific characteristics like older age or comorbidities. Patients not meeting these criteria can also be treated in a hospital without burn centre. Limited information is available about the organisation of care and referral of these patients. The aims of this study are to determine the burn injury characteristics, treatment (costs), quality of life and scar quality of burn patients admitted to a hospital without dedicated burn centre. These data will subsequently be compared with data from patients with $<10 \%$ total bodysurface area (TBSA) burned who are admitted (or secondarily referred) to a burn centre. If admissions were in agreement with the EMSB, referral criteria will also be determined.

Methods and analysis In this multicentre, prospective, observational study (cohort study), the following two groups of patients will be followed: 1) all patients (no age limit) admitted with burn-related injuries to a hospital without a dedicated burn centre in the Southwest Netherlands or Brabant Trauma Region and 2) all patients (no age limit) with $<10 \%$ TBSA burned who are primarily admitted (or secondarily referred) to the burn centre of Maasstad Hospital. Data on the burn injury characteristics (primary outcome), EMSB compliance, treatment, treatment costs and outcome will be collected from the patients' medical files. At 3 weeks and at 3, 6 and 12 months after trauma, patients will be asked to complete the quality of life questionnaire (EuroQoL-5D), and the patient-reported part of the Patient and Observer Scar Assessment Scale (POSAS). At those time visits, the coordinating investigator or research assistant will complete the observer-reported part of the POSAS.

\section{Strengths and limitations of this study}

- This study will provide insight into care of burn patients both in hospitals with and without a dedicated burn centre.

- It is a prospective, multicentre, observational study with a best possible methodological design.

- Participation of over 20 hospitals will increase the reliability and generalisability of the data.

- Although the study will be mostly relevant for the Netherlands, it is also informative for other regions.

Ethics and dissemination This study has been exempted by the medical research ethics committee Erasmus MC (Rotterdam, The Netherlands). Each participant will provide written consent to participate and remain encoded during the study. The results of the study are planned to be published in an international, peer-reviewed journal. Trial registration number NTR6565.

\section{INTRODUCTION}

Burns cause significant morbidity and mortality worldwide. Globally, some studies are available on the epidemiology of emergency department (ED) treatments of burn injuries, ${ }^{1-8}$ including three from the Netherlands. ${ }^{7-9}$ These data were limited to home and leisure accidents; work-related burn injuries were not included.

Depending on the severity, burn injuries may require specialised burn care. In order to enable proper triaging and referral, 
Box 1 Emergency Management of Severe Burns referral criteria $^{10}$

1. The percentage total body surface area burned (TBSA burned; $>10 \%$ TBSA in adults or $>5 \%$ in children).

2. Burns of special areas (face, hands, feet, perineum, genitalia and major joints).

3. Full thickness burns $>5 \%$ TBSA burned.

4. Electrical burns.

5. Chemical burns.

6. Burns with associated inhalation injury.

7. Circumferential burns of limbs or chest.

8. Extremes of age (children and elderly).

9. Pre-existing medical disorders, which could complicate management and prolong recovery or effect mortality.

10. Associated trauma.

the Emergency Management of Severe Burns (EMSB) referral criteria have been implemented (box 1). ${ }^{10}$

In 2014, 322 patients with burns or inhalation injury were admitted to a hospital in the trauma region of Southwest Netherlands. Of these patients, 109 (34\%) were admitted to hospitals without specialised burn centre. There is a lack of insight into the epidemiology, treatment (including treatment costs) and outcome (both clinical results and quality of life) of the patients treated in hospitals without burn centre.

In order to get insight into the epidemiology of burn patients, detailed data collection on patient characteristics, aetiology, burn injury characteristics, treatment and outcome is critical. Such data are currently not available from current registries. The Dutch Burn Repository R3 only registers data for patients admitted to a burn centre. ${ }^{11}$ The National Injury Surveillance System (in Dutch: Letsel Informatie Systeem; www.veiligheid. $\mathrm{nl})^{12}$ and the National Medical Registration (in Dutch: Landelijke Medische Registratie; www.dutchhospitaldata. nl) ${ }^{13}$ register non-admitted and admitted patients, respectively. Both are insufficient, as they register patients based on the main diagnosis, which may not always be the burn injury. The Dutch National Trauma Registry (in Dutch: Landelijke Trauma Registratie; www.lnaz.nl) registers all admitted trauma patients. It encodes burn wounds, but with limited detail, and does not contain relevant treatment and outcome data.

Based on the above, there are currently no data available that provide sufficient details about patients admitted to a hospital without burn centre. It is also not clear if these are different for patients with similar burn injury severity admitted to a burn centre. The main aim of this study is to determine the burn injury characteristics of burn patients admitted to a hospital without dedicated burn centre. Secondary aims are 1) to determine if these admissions were in agreement with the EMSB referral criteria; 2) to determine the treatment and direct medical costs, the quality of life (EuroQoL-5D (EQ-5D)) and scar quality (Patient and Observer Scar Assessment Scale (POSAS)) for these patients until 12 months follow-up and 3) to compare the injury pattern and outcome of these patients with burn patients with $<10 \%$ total body surface area (TBSA) burned who are admitted (or secondarily referred) to a burn centre. We expect that burn patient admission outside the burn centre is restricted to a maximum of $10 \%$ TBSA, and that outcome is similar to that achieved for a subgroup with similar burn injury severity admitted to a burn centre.

\section{METHODS AND ANALYSES \\ Study design and setting}

This study will follow a multicentre, prospective, observational study design (cohort study). Patients will be recruited from every hospital in two large trauma regions in The Netherlands, the Southwest Netherlands trauma region and Network Emergency Care Brabant. The Burn Centre of Maasstad Hospital is included in the Southwest Netherlands trauma area. The following 18 hospitals in The Netherlands will participate: Admiraal De Ruyter Ziekenhuis (Goes), Albert Schweitzer Ziekenhuis (Dordrecht), Amphia Ziekenhuis (Breda), Beatrix Ziekenhuis (Gorinchem), Bravis Ziekenhuis (Roosendaal), Catharina Ziekenhuis (Eindhoven), Elisabeth-TweeSteden Ziekenhuis (Tilburg), Elkerliek Ziekenhuis (Helmond), Erasmus MC, University Medical Centre (Rotterdam), Franciscus Gasthuis and Vlietland (Rotterdam), IJsselland Ziekenhuis (Capelle aan de IJssel), Ikazia Ziekenhuis (Rotterdam), Jeroen Bosch Ziekenhuis ('s-Hertogenbosch), Burn Centre Maasstad Hospital (Rotterdam), Máxima Medisch Centrum (Veldhoven), Van Weel-Bethesda Ziekenhuis (Dirksland) and ZorgSaam Zeeuws-Vlaanderen (Terneuzen). The study started in September 2017 and the recruiting periods will be 18 months. With a follow-up of 1 year, the presentation of data will be expected by the end of 2020 .

\section{Study registration}

The study is registered at the Netherlands Trial Register (NTR6565; 12 July 2017).

\section{Recruitment and informed consent}

Eligible persons admitted with burn-related injuries (or the parents of paediatric patients) will be informed about the study as soon as possible after hospital presentation. They may receive the information at the ED or while admitted at the surgical ward. The local staff will ask permission to send contact details to the research team. On receipt of that permission, a local contact person in the participating hospital will provide details of the patient to the research team. The coordinating investigator or research assistant will contact the patient (or parents) to explain the study, and will send the information brochure and informed consent form. The coordinating investigator or a research assistant will attend the patient's outpatient visit in the hospital (at approximately 3 weeks after admission) in order to further explain the study, answer any question the patient (or parents) 
may have and for signing informed consent. This gives patients on average 2-3 weeks to consider their participation. As data collection also includes investigation of the TBSA and extent of burn wounds, it is not possible to give patients more time to consider their participation.

In order to reduce bias as much as possible, the follow-up measurements by the clinical investigator or research assistant will be performed using a standardised protocol.

\section{Study population and eligibility criteria}

The study population will consist of a group of patients admitted to a hospital without dedicated burn centre or who are admitted to a dedicated burn centre. In order to be eligible to participate in this study, a subject must meet all of the following criteria:

1. Patients with burn-related injuries (no age limit), admitted to a hospital without dedicated burn centre in the trauma regions of Southwest Netherlands or Brabant; or patients with $<10 \%$ TBSA burned, who are primarily admitted or secondarily referred to the burn centre of Maasstad Hospital. ${ }^{i}$

2. Provision of informed consent.

A potential subject who meets any of the following criteria will be excluded from participation in this study:

1. Patients who died $<24$ hours due to severity of nonburn injuries (eg, severe head injury).

2. Patients with incomplete or unknown contact information.

3. Insufficient comprehension of the Dutch or English language to understand the questionnaires.

\section{Outcome measures}

The burn-related injury pattern will serve as primary outcome measure. Information on the following items, representing injuries and mechanism, will be collected from the patients' medical files:

- Inhalation injury (yes or no).

- Body regions burned, severity (\% TBSA burned as assessed by a trained research physician).

- Extent of burns (superficial dermal, deep dermal or subdermal).

- Setting (home, work or other).

- Burn aetiology (scald, flame, contact, chemical or other).

The secondary outcome measures are:

1. Compliance with EMSB referral criteria (yes or no).

2. Treatment.

3. Healthcare use in hospital with associated treatment costs.

4. Health-related quality of life (EuroQol-5D-3L (EQ-5D3L)).

5. POSAS.

${ }^{\mathrm{i}}$ Ten per cent has been chosen as we expect that patients admitted outside the burn centre will have no more that $10 \%$ TBSA burned. This is also in line with the EMSB referral criteria. ${ }^{10}$ The \% TBSA will be assessed during physical examination by a research physician who has had elaborate training for this at the participating dedicated burn centre.
The following treatment characteristics will be collected:

- Prehospital transport (ambulance, helicopter emergency medical services, or other).

- Level of trauma care (level 1, 2 or 3).

- Required care (ward or intensive care unit).

- Resuscitation (amount of fluid <24hours after admission).

- Intubation (yes or no).

- Bronchoscopy (yes or no).

- Wound treatment (wound dressing, topical therapy or other).

- Date of the first operation.

- Escharotomy (yes or no).

- Type of operation (split skin grafting, full-thickness skin grafting or excision and primary closure).

- \% TBSA that needed skin grafting.

- Expansion of split skin graft (1:1, 1:1.5, 1:3 or 1:6).

- Total number of operations.

- Indication for second, third and any subsequent operation.

- Number of outpatient department visits.

- Date of discharge from hospital.

- Reconstructive surgery (release/excision with split skin grafting, full-thickness grafting or laps).

In order to determine the treatment costs, the use of hospital resources will be collected from medical files directly. All direct medical costs due to treatment, complications and events during follow-up (eg, ED visit, diagnostic workup, therapy, events, surgery, admissions, follow-up visits) will be collected. The economic evaluation will be in accordance with the Dutch guideline and will use cost prices where possible. ${ }^{14}$ Medical costs will be calculated by multiplying the volumes of healthcare use with the corresponding unit prices.

The EQ-5D is a validated questionnaire for measuring health-related quality of life. ${ }^{1516}$ Its use is recommended for the assessment of quality of life in trauma patients, especially for economic assessments. ${ }^{17} 18$ The EQ-5D-3L descriptive system consists of five dimensions of health (mobility, self-care, usual activities, pain/discomfort anxiety/depression), each with three possible answers. Scores are converted to a utility score ranging from zero to one, with lower scores indicating poorer quality of life. The EQ Visual Analogue Score records the respondents self-rated health status on a vertical $(0-100)$ visual analogue scale. Patients aged 16 years or older will be asked to complete the EQ-5D themselves. Paediatric patients $(<16$ years) will be asked to compete the youth version of the EQ-5D, or their parent(s) will be asked to complete a proxy version of the EQ-5D youth version.

The POSAS V.2.0 (www.posas.org) ${ }^{19}$ consists of two parts: a patient scale and an observer scale, which aim to provide a rating of several measured of scar quality (vascularity, pigmentation, relief/texture, thickness, pliability, surface area, pain and itching/pruritus). Patients aged 16 years or older and parents of younger paediatric patients will complete the patient-reported part, a trained observer will complete the observer-reported part. 


\section{Other data collected}

The following additional data will be collected in order to describe the study population, patient characteristics, additional injury characteristics and burn-related (adverse) events:

- Patient characteristics: age, gender, American Society of Anesthesiologists classification, comorbidities (diabetes or other).

- Additional injury characteristics: Injury Severity Score, Abbreviated Injury Score, for all nine anatomical regions as registered in the trauma registry).

- Burn-related (adverse) events: hematoma (yes or no), excessive blood loss (requiring blood transfusion), wound infection (requiring antibiotic and/or surgical treatment) (yes or no), pneumonia (yes or no), graft loss requiring surgical intervention (or vac therapy; yes or no) or other.

- Outcome-related variables: duration of hospital admission, time to wound closure (calculated from date of admission and date of last wound dressing), percentage graft take 5-7 days postburn, discharge destination (home, other hospital, burn centre, rehabilitation facility or other) and mortality (possibly burn-related; yes or no).

\section{Study procedures, data collection methods and participant} timelines

Patients will be followed for 1 year, with visits planned at 3 weeks (window 2-4 weeks), 3 months (window 11-15 weeks), 6 months (window 5-7 months) and 12 months (window 12-14 months) after admission (table 1). One additional, optional visit is planned at 6 weeks (window 5-7 weeks) in case the burn wound has not closed at 3 weeks. Wound closure is defined as 95\% closure, as judged on wound inspection by the research physician and the local treating physician or specialised wound care nurse. In case of disagreement, consensus will be reached by discussion. The coordinating investigator or research assistant will be present at each outpatient department visit of the enrolled patients for direct collection of data. The study will not interfere with treatment or follow-up.

Patient characteristics, injury characteristics and any other relevant data will be collected from the patient's medical files as soon as possible after signing informed consent.

Treatment and other clinical data such as adverse events will be collected (partly from the patient files) at each follow-up visit. During these regular clinical visits, any radiographs or digital photos that are routinely obtained will be collected. For the purpose of the study, additional details concerning treatment and outcome that are not mentioned in the patient's hospital files, may be registered on the case report forms directly. At the 1-year follow-up visit, the coordinating investigator or research assistant will document any secondary intervention that may be planned for the patient.

As soon as possible after inclusion, patients will be asked to complete the EQ-5D-3L reflecting their preinjury quality of life. At 3, 6 and at 12 months after the primary hospital admission, patients (or the parents of paediatric patients) will be asked to complete the EQ-5D-3L and the patient-reported part of the POSAS. The coordinating

Table 1 Schedule of events

\begin{tabular}{|c|c|c|c|c|c|c|}
\hline & & 3 weeks & 6 weeks* $^{*}$ & 3 months & 6 months & 12 months \\
\hline Event forms & Screening & (2-4 weeks) & (5-7 weeks) & (11-15 weeks) & (6-7 months) & (12-14 months) \\
\hline Screening & $x$ & & & & & \\
\hline Informed consent & & $x$ & & & & \\
\hline Patient characteristics & & $x$ & & & & \\
\hline Injury characteristics & & $x$ & & & & \\
\hline Radiology & & $x+$ & $x+$ & $x+$ & $\mathrm{x} \dagger$ & $x+$ \\
\hline Digital photo & & $x+$ & $x \dagger$ & $x \dagger$ & $x \dagger$ & $x \dagger$ \\
\hline Treatment characteristics & & $x$ & $x$ & $x$ & $x$ & $x$ \\
\hline Outcome details & & $x$ & $x$ & $x$ & $x$ & $x$ \\
\hline Adverse events & & $x$ & $x$ & $x$ & $x$ & $x$ \\
\hline Clinical FU & & $x$ & $x$ & $x$ & $x$ & $x$ \\
\hline Healthcare consumption & & $x$ & $x$ & $x$ & $x$ & $x$ \\
\hline$E Q-5 D$ & & $X \ddagger$ & & $x$ & $x$ & $x$ \\
\hline POSAS & & & & $x$ & $x$ & $x$ \\
\hline Early withdrawal & & $\S$ & $\S$ & $\S$ & $\S$ & $\S$ \\
\hline
\end{tabular}

*Optional visit; will only take place if wounds are not closed at 3 weeks.

†Only if treating surgeon requests these images.

$\ddagger$ Asking for EQ-5D preburn.

§Only at time of withdrawal.

EQ-5D, EuroQoL-5D; FU, follow-up; POSAS, Patient and Observer Scar Assessment Scale. 
investigator or research assistant will complete the physician-reported part of the POSAS.

\section{Blinding}

Patients, the research physician who determines \% TBSA, wound closure and scar quality, and the statistician will not be blinded.

\section{Sample size}

Given the epidemiological nature of the primary aim and the lack of information of the population admitted to a hospital without burn centre, a formal sample size calculation was not made. By including all patients treated in the trauma regions of Southwest Netherlands and Brabant during 18 months, as well as all patients with $<10 \%$ TBSA burned who are primarily admitted or secondarily referred to a burn centre, the maximum number of inclusions will be achieved in both cohorts. The larger the number of patients, the more reliable the analysis will be. Enrolling all patients possible will result in the highest reliability of data (ie, accurate point estimate with lowest possible variance). Based on the data in the annual report of Trauma Centre Southwest Netherland (ie, 109 patients admitted outside the burn centre in the year 2014), we expect to include approximately 300 patients in 18 months in the two trauma regions combined.

\section{Statistical analysis}

Data will be analysed using the Statistical Package for the Social Sciences (SPSS) V.21.0 or higher (SPSS, Chicago, Illinois, USA) and will be reported following the Strengthening the Reporting of Observational studies in Epidemiology guidelines. Missing values will not be replaced by imputation. Normality of continuous data will be tested with the Shapiro-Wilk test, and homogeneity of variances will be tested using the Levene's test. A p value $<0.05$ will be taken as threshold of statistical significance in all statistical tests, and all tests will be two-sided. No interim analysis is planned.

First, descriptive analysis will be performed in order to report the outcome measures and other collected data. Data will be reported for the entire population as well as separately for the group of patients admitted to a hospital without versus with a burn centre. For continuous data (eg, EQ-5D and POSAS), the mean and SD (parametric data) or the median and quartiles (non-parametric data) will be reported. The only exception are cost data, these will be reported as mean with $95 \%$ CI. The $95 \%$ CI around the mean costs will be approximated by non-parametric bootstrapping. For categorical data (eg, EMSB compliance), the number and frequencies will be reported.

Next univariate analysis will be done in order to compare the two cohorts. Statistical significance of difference between the two groups will be tested using a Student's t-test (parametric, continuous data; with or without equal variance assumed as applicable), Mann-Whitney U test (non-parametric, continuous data) or $\mathrm{X}^{2}$ test or Fisher's exact test (categorical data, as applicable).
Subgroups will be determined based on the patients included. Relevant subgroups can be children versus adults versus elderly, subgroups with different percentages TBSA burned or patients admitted in compliance versus disagreement with the EMSB referral criteria. Should formal testing between the latter two subgroups be feasible, this will be done as described above.

The economic evaluation will include costs for healthcare. Direct and indirect, medical and non-medical cost will be measured as indicated in the Dutch guidelines for economic evaluations, using standard, published cost prices where possible. $^{14}$

\section{Data management and monitoring}

Data will be encoded and stored in a pass-word protected OpenClinica database with restricted access to the researchers only. Data will be entered once. Quality of the entered data will be monitored by checking entry for a random sample of patients prior to database locking.

\section{Patient and public involvement}

The need for this study emerged from meetings of the Association of Dutch Burn Centres as well as the Dutch Burns Foundation, representing both healthcare professionals and patients. Input into the design, comparison and outcome measures was given during presentation for the Scientific Board of the Dutch Burns Foundation. Patients were not directly involved in the subsequent writing of the protocol, nor were they directly involved in the recruitment to and conduct of the study. A summary of the main results will be made available to study participants on request.

\section{Ethics and dissemination}

This study will be conducted according to the principles of the Declaration of Helsinki (64th World Medical Association General Assembly, Fortaleza, Brazil, October 2013).

Approval has been obtained from the local hospital boards in all participating centres. Following review of the protocol (V.1.0, 22 May 2017), the MREC concluded that this study is not subject to the Medical Research Involving Human Subjects Act (WMO). They concluded that the study is a medical/scientific research, but no patients are subjected to procedures or are required to follow rules of behaviour. Consequently, the statutory obligation to provide insurance for subjects participating in medical research (article 7 of the WMO) does not apply. Any important changes in the protocol will be submitted to the accredited MREC. The results of the study are planned to be published in an international, peer-reviewed journal and presented at national and international trauma and burn care meetings. Each participating centre will provide a group author to every publication. No personal data of study participants will be presented.

\section{DISCUSSION}

Burns cause significant morbidity and mortality worldwide, and depending on the severity, burn injuries may 
require specialised burn care in a dedicated burn centre. In order to enable optimal triaging and referral to a burn centre, the EMSB referral criteria have been implemented. ${ }^{10}$ Whereas extensive injury and outcome registration exists for patients admitted to a dedicated burn centre, there are currently no data available that provide sufficient details about patients admitted to a hospital without burn centre. Successful completion of this study will provide more insight into the percentage of patients admitted to a hospital without dedicated burn centre. It will also provide us detailed data on patient and injury characteristics, EMSB compliance, treatment, treatment costs, relevant outcome data and quality of life of burn patients with primary admission at a hospital without burn centre as well as patients with $<10 \%$ TBSA burned who are secondarily referred to a burn centre.

Twenty-one hospitals will participate. Although the participation of multiple hospitals may introduce treatment bias, it also makes the results more generalisable.

\section{Author affiliations}

${ }^{1}$ Trauma Research Unit Department of Surgery, University Medical Center Rotterdam, Rotterdam, The Netherlands

${ }^{2}$ Burn Center, Maasstad Hospital, Rotterdam, Zuid-Holland, The Netherlands ${ }^{3}$ Association of Dutch Burn Centers, Maasstad Hospital, Rotterdam, Zuid-Holland, The Netherlands

${ }^{4}$ Department of Public Health, Erasmus MC, University Medical Center Rotterdam, Rotterdam, Zuid-Holland, The Netherlands

${ }^{5}$ Department of Surgery, Jeroen Bosch Ziekenhuis, Den Bosch, Noord-Brabant, The Netherlands

${ }^{6}$ Department of Surgery, ZorgSaam Zeeuws-Vlaanderen, Terneuzen, Zeeland, The Netherlands

${ }^{7}$ Department of Surgery, IJsselland Ziekenhuis, Capelle aan den IJssel, ZuidHolland, The Netherlands

${ }^{8}$ Department of Surgery, Elkerliek Ziekenhuis, Helmond, The Netherlands

${ }^{9}$ Department of Surgery, Franciscus Gasthuis\&Vlietland, Rotterdam, Zuid-Holland, The Netherlands

${ }^{10}$ Department of Surgery, Elisabeth-TweeSteden Ziekenhuis, Tilburg, Noord-Brabant, The Netherlands

${ }^{11}$ Department of Surgery, Ikazia Ziekenhuis, Rotterdam, Zuid-Holland, The Netherlands

${ }^{12}$ Department of Surgery, Van Weel-Bethesda Ziekenhuis, Middelharnis, ZuidHolland, The Netherlands

${ }^{13}$ Department of Surgery, Beatrix Ziekenhuis/Rivas, Gorinchem, Zuid-Holland, The Netherlands

${ }^{14}$ Department of Surgery, Catharina Ziekenhuis, Eindhoven, Noord-Brabant, The Netherlands

${ }^{15}$ Department of Surgery, Bravis Ziekenhuis, Bergen op Zoom, Noord-Brabant, The Netherlands

${ }^{16}$ Department of Surgery, Máxima Medisch Centrum, Veldhoven, Noord-Brabant, The Netherlands

${ }^{17}$ Department of Surgery, Albert Schweitzer Ziekenhuis, Dordrecht, Zuid-Holland, The Netherlands

${ }^{18}$ Department of Surgery, Amphia Ziekenhuis, Breda, The Netherlands

${ }^{19}$ Department of Surgery, Admiraal De Ruyter Ziekenhuis, Goes, Zeeland, The Netherlands

Contributors EMMVL, DTVY, MEVB, SP, MHJV and CHVdV developed the study. MHJV will act as trial principal investigator. DTVY, DB, AYMVPC, PARDR, MG, TMALK, KWWL, ANR, MS, LVdS, AHvdV, FCVE, PVVE, PAV, DIV, MW, MHJV and CHVdV will participate in patient inclusion and outcome assessment. DTVY, SP and EMMVL will perform statistical analysis of the study data. DTVY and EMMVL drafted the current manuscript. All other authors have read and approved this final manuscript. At the end of the study, DTVY and EMMVL will draft the manuscript. All other authors will interpret the data, critically revise the manuscript and approve the final version to be submitted.

Funding This research received a specific grant from the Dutch Burns Foundation (Beverwijk, The Netherlands; reference number W0/16.110).

Disclaimer The funder of the study has no role in study design, data collection, data analysis, data interpretation or writing of the report.

Competing interests None declared.

Patient consent Not required.

Ethics approval This study was approved by the medical research ethics committee (MREC) Erasmus MC (Rotterdam, The Netherlands) (reference number MEC-2017-356).

Provenance and peer review Not commissioned; externally peer reviewed.

Open access This is an open access article distributed in accordance with the Creative Commons Attribution Non Commercial (CC BY-NC 4.0) license, which permits others to distribute, remix, adapt, build upon this work non-commercially, and license their derivative works on different terms, provided the original work is properly cited, appropriate credit is given, any changes made indicated, and the use is non-commercial. See: http://creativecommons.org/licenses/by-nc/4.0/.

\section{REFERENCES}

1. Akerlund $E$, Huss FR, Sjöberg F. Burns in Sweden: an analysis of 24,538 cases during the period 1987-2004. Burns 2007:33:31-6.

2. DeKoning EP, Hakenewerth A, Platts-Mills TF, et al. Epidemiology of burn injuries presenting to North Carolina emergency departments in 2006-2007. Burns 2009;35:776-82.

3. D'Souza AL, Nelson NG, McKenzie LB. Pediatric burn injuries treated in US emergency departments between 1990 and 2006. Pediatrics 2009;124:1424-30.

4. Fagenholz PJ, Sheridan RL, Harris NS, et al. National study of Emergency Department visits for burn injuries, 1993 to 2004. J Burn Care Res 2007;28:681-90.

5. Onarheim H, Jensen SA, Rosenberg BE, et al. The epidemiology of patients with burn injuries admitted to Norwegian hospitals in 2007. Burns 2009;35:1142-6.

6. Wasiak J, Spinks A, Ashby K, et al. The epidemiology of burn injuries in an Australian setting, 2000-2006. Burns 2009;35:1124-32.

7. den Hertog PC, Blankendaal FA, ten Hag SM. Burn injuries in The Netherlands. Accid Anal Prev 2000:32:355-64.

8. van Rijn OJ, Grol ME, Bouter LM, et al. Incidence of medically treated burns in The Netherlands. Burns 1991;17:357-62.

9. Goei H, Wijnen BFM, Mans S, et al. Optimizing recruitment strategy for follow-up in patients with a burn related emergency department visit: a feasibility study. submitted.

10. The Education Committee of the Australian and New Zealand Burn Association. Emergency management of severe burns (EMSB) course manual, Dutch version: Dutch Burn Foundation, 2009.

11. Dokter J, Vloemans AF, Beerthuizen Gl, et al. Epidemiology and trends in severe burns in the Netherlands. Burns 2014;40:1406-14.

12. Meerding WJ, Polinder S, Lyons RA, et al. How adequate are emergency department home and leisure injury surveillance systems for cross-country comparisons in Europe? Int J Inj Contr Saf Promot 2010;17:13-22.

13. Van der Stegen R, Ploemacher J. Description of methods for statistics by diagnoses in time by using the LMR (1981-2005. The Hague: Statistics Netherlands, 2009;9.

14. Hakkaart-van Roijen L, Van der Linden N, Bouwmans C, et al. Tan SS Van goede zorg verzekerd, BIJLAGE 1: Kostenhandleiding: Methodologie van kostenonderzoek en referentieprijzen voor economische evaluaties in de gezondheidszorg, 2016.

15. Brooks R, Rabin RE, eds. DC: The measurement and valuation of health status using EQ-5D: a European perspective. Europe: Kluwer Academic Publishers, 2003.

16. Lamers LM, Stalmeier PF, McDonnell J, et al. [Measuring the quality of life in economic evaluations: the Dutch EQ-5D tariff]. Ned Tijdschr Geneeskd 2005;149:1574-8.

17. Neugebauer E, Bouillon B, Bullinger M, et al. Quality of life after multiple trauma-summary and recommendations of the consensus conference. Restor Neurol Neurosci 2002;20(3-4):161-7.

18. Van Beeck EF, Larsen CF, Lyons RA, et al. Guidelines for the conduction of follow-up studies measuring injury-related disability. $J$ Trauma 2007;62:534-50.

19. van de Kar AL, Corion LU, Smeulders MJ, et al. Reliable and feasible evaluation of linear scars by the patient and observer scar assessment scale. Plast Reconstr Surg 2005;116:514-22. 